\title{
The Effect of Sodium Hyaluronate on Ligamentation and Biomechanical Property of Tendon in Repair of Achilles Tendon Defect with Polyethylene Terephthalate Artificial Ligament: A Rabbit Tendon Repair Model
}

\author{
Shengkun Li, Kui Ma, Hong Li, Jia Jiang, and Shiyi Chen \\ Department of Sports Medicine, Huashan Hospital, 12 Wulumuqi Zhong Road, Shanghai 200040, China \\ Correspondence should be addressed to Hong Li; lihongdr@163.com
}

Received 30 June 2016; Revised 16 October 2016; Accepted 1 November 2016

Academic Editor: George Babis

Copyright (C) 2016 Shengkun Li et al. This is an open access article distributed under the Creative Commons Attribution License, which permits unrestricted use, distribution, and reproduction in any medium, provided the original work is properly cited.

The Achilles tendon is the most common ruptured tendon of human body. Reconstruction with polyethylene terephthalate (PET) artificial ligament is recommended in some serious cases. Sodium hyaluronate (HA) is beneficial for the healing of tendon injuries. We aimed to determine the effect of sodium hyaluronate in repair of Achilles tendon defect with PET artificial ligament in an animal tendon repair model. Sixteen New Zealand White rabbits were divided into two groups. Eight rabbits repaired with PET were assigned to PET group; the other eight rabbits repaired with PET along with injection of HE were assigned to HA-PET group. All rabbits were sacrificed at 4 and 8 weeks postoperatively for biomechanical and histological examination. The HA-PET group revealed higher biomechanical property compared with the PET group. Histologically, more collagen tissues grew into the HA-PET group compared with PET group. In conclusion, application of sodium hyaluronate can improve the healing of Achilles tendon reconstruction with polyethylene terephthalate artificial ligament.

\section{Introduction}

The incidence of Achilles tendon rupture increased during the past few years [1]. In some serious cases, direct repair is impossible. In that case, reconstruction of the tendon is required. There are many methods to treat the Achilles tendon rupture, including autograft reconstruction $[2,3]$, allograft reconstruction [4], and artificial ligament reconstruction [5]. However, the autograft tendon has the donor site problem, while the allograft tendon may increase the risk of disease transfusion or immunological rejection.

Artificial ligament is frequently used in the reconstruction of ligament rupture management like anterior cruciate ligament reconstruction [6], acromioclavicular joint reconstruction [7], also the Achilles tendon reconstruction [5], etc. Artificial ligaments can promise a fast functional recovery and avoid complications. In addition, there are various treatments applied for helping the healing of tendon injuries such as platelet-rich plasma (PRP) $[8,9]$ and sodium hyaluronate (HA) [10]. Sodium hyaluronate (HA) is polysaccharide, which is found in all extracellular matrix of vertebrates and in some bacteria as well [11]. In this way, it may be helpful to the wound healing process by generating proper environment for growth, leading to the accumulation of several matrix proteins [12].

Based on the background before, we applied the sodium hyaluronate (Bausch \& Lomb Freda Co., Ltd., Shandong, China) after the repair procedure of Achilles tendon defect with PET artificial ligament. The aim of this study was to analyze the effect of sodium hyaluronate on ligamentation and biomechanical property of tendon in repair of Achilles tendon defect with PET in a rabbit tendon repair model.

\section{Materials and Methods}

2.1. Experimental Design. The animal experiment was approved by the Animal Care and Use Committee of our college. Two groups were involved in this study; the group 


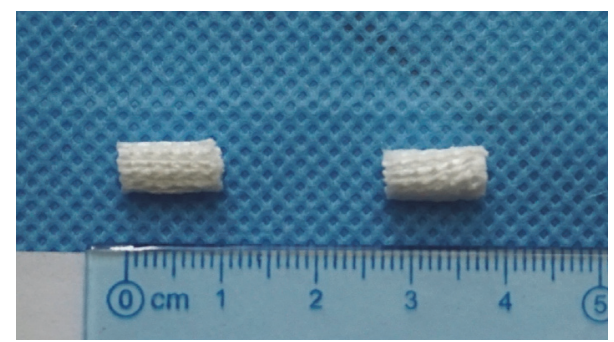

FIGURE 1: Optical photograph of artificial ligament graft.

treated with sodium hyaluronate (HA) was set as an experimental group, while another group untreated with sodium hyaluronate was prepared as a control group.

2.2. Animals. Total 16 fifteen-week-old male New Zealand White rabbits (mean weight $2.6 \pm 0.2 \mathrm{~kg}$ ) were randomly selected for experiments. In the study, 8 rabbits were assigned to the experimental group, while another 8 rabbits were used for the control group. Animals were sacrificed at the time points of weeks $4(n=4)$ and $8(n=4)$ after surgery for both the experimental group and the control group.

2.3. Preparation of PET Sheet. PET sheets (Figure 1) were taken from the Ligament Advanced Reinforcement System (LARS) ligament and then soaked in $75 \%$ alcohol solution to remove foreign material and contamination for 4 hours, cleaned with a large amount of deionized water and then dried in air for 24 hours $[13,14]$.

2.4. Operative Procedure. Anesthesia was induced by the intravenous administration of $3 \%$ pentobarbital $(30 \mathrm{mg} / \mathrm{kg})$ [13]. The animal was placed prone on the operating table. After preparation of skin and disinfection, a longitudinal skin incision was made. Then, two-thirds of vertical plane of the Achilles tendon was cut by scissors to create a partial tendon injury. To help the Achilles tendon to heal, the PET sheets were implanted into the defect to strengthen the tendon defect with 5-0 sutures in the experimental group with the injection of $2 \mathrm{ml}$ sodium hyaluronate $(10 \mathrm{mg} / \mathrm{ml})$ over the surface of the tendon defect before the skin incision was closed routinely [13]. The control group was performed in the same way without the injection of sodium hyaluronate. The animals were sent back to their cages and allowed free cage activity without immobilization after surgery. All animals were given buprenorphine $(0.05 \mathrm{mg} / \mathrm{kg})$ subcutaneously for 3 days for pain control [15]. The rabbits were, respectively, sacrificed at weeks 4 and 8 after surgery to harvest tissue specimens for histological and biomechanical analysis.

2.5. Histological Analysis. Identically located longitudinal tendon samples ( $n=1$ tendon of each group at each time) were collected randomly from the injured and normal tendons [16]. After fixation in $10 \%$ formalin for $24 \mathrm{~h}$, the tendon samples were washed, dehydrated, cleared, and embedded in paraffin wax. The samples were then sectioned into layers with a thickness of $5 \mathrm{um}$ perpendicular to the longitudinal axis of the tendon using a microtome (SM2500, Leica) [17]. These sections were stained with hematoxylin and eosin (H\&E) staining and Masson trichrome staining [18]. The slides were examined by light microscopy (Olympus Optical Co., Tokyo, Japan) for initial evaluation and photography $[13,16-18]$. Two evaluators who performed the histological observation were blinded to treatment group of the sections.

2.6. Biomechanical Analysis. The repaired tendons $(n=3$ tendons of each group at each time) were harvested after sacrifice and subjected to mechanical testing within $6 \mathrm{~h}$, using a biomechanical analyzer (AGS-X $5 \mathrm{kN}$, Shimadzu Co., Japan). The dimension of the test tendon sample is approximately $4 \mathrm{~cm}$. And all the samples were prepared for testing without being frozen. Both ends of the tendon were sutured by number 5 Ethibond suture for traction. Then, the sutures were fixed firmly in the biomechanical analyzer. Care was taken to keep the longitudinal tendon samples parallel to the testing axis. After preconditioning, the ultimate load-to-failure was performed with the extension rate of $5 \mathrm{~mm} / \mathrm{min}$. The load-to-failure $(\mathrm{N})$ and the stiffness $(\mathrm{N} / \mathrm{mm})$ were measured, while the load-distortion curve was recorded. For each sample, testing ends when the tendon ruptured.

2.7. Statistical Analysis. Results are presented as mean and standard deviation. The paired Student $t$-test was used to compare the experimental group with control group to determine the significant differences. If $p<0.05$, the differences were considered significant statistically.

\section{Results}

3.1. Mechanical Examination Results. No tendon rupture occurred for all specimens of both 4 and 8 weeks. The most common failure mode is the slippage of rope from the test tendon. At 4 weeks, there was no statistically significant difference in the load-to-failure between the HA-PET group and the control group $(171.7 \pm 38.1 \mathrm{~N}$ for HA-PET group and $156.7 \pm 12.7 \mathrm{~N}$ for controls; $p>0.05$ ), while at 8 weeks there was also no statistically significant difference between two groups (175.3 $\pm 44.7 \mathrm{~N}$ for HA-PET group and $137 \pm 65.8 \mathrm{~N}$ for controls; $p>0.05$ ) (Figure 2). Similarly, at 4 weeks there was no statistically significant difference in the stiffness between the HA-PET group and the control group $(4.2 \pm 2.4 \mathrm{~N} / \mathrm{mm}$ for HA-PET group and $2.1 \pm 0.9 \mathrm{~N} / \mathrm{mm}$ for controls; $p>$ 0.05). Meanwhile at 8 weeks there was also no statistically significant about stiffness difference between two groups $(4.5$ $\pm 3.8 \mathrm{~N} / \mathrm{mm}$ for HA-PET group and $2.1 \pm 0.3 \mathrm{~N} / \mathrm{mm}$ for controls; $p>0.05$ ) (Figure 3).

3.2. Histological Results. Four weeks after surgery it appeared that there was no tissue surrounding the graft fibers in both the PET and HA-PET groups. After 8 weeks, thick collagen tissue with some vasculature covered the grafts in the HAPET group, while there was little tissue infiltration of the graft fibers in the PET group (Figures 4 and 5). Some cells from the fibrous tissue infiltrated the graft site, and the collagen fibers tended to orient along the axis of the tendon. 


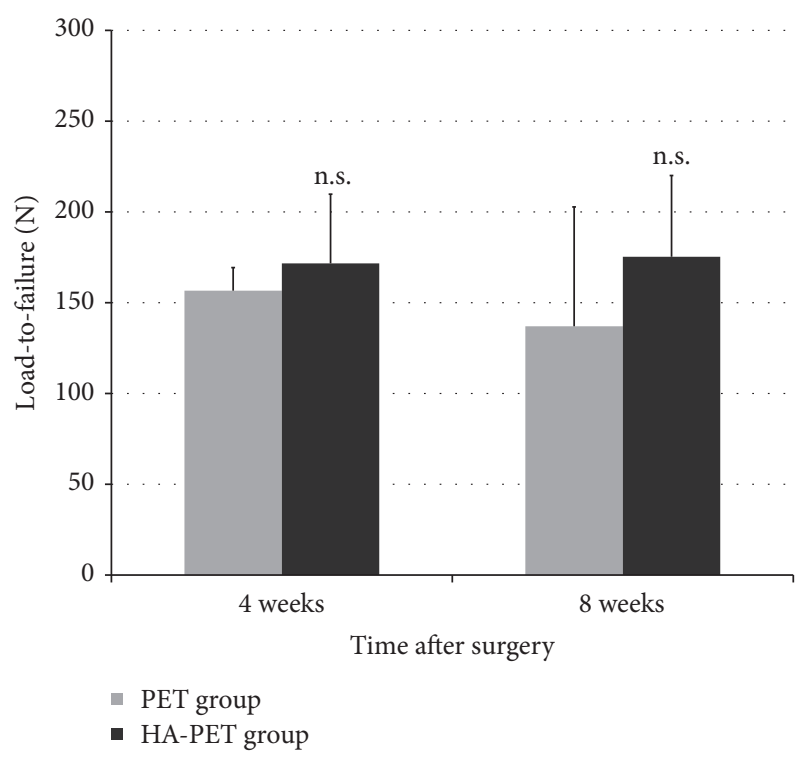

FIGURE 2: Comparison of maximal failure load for Achilles tendon healing in a rabbit model of the PET group and HA-PET group at each time after surgery.

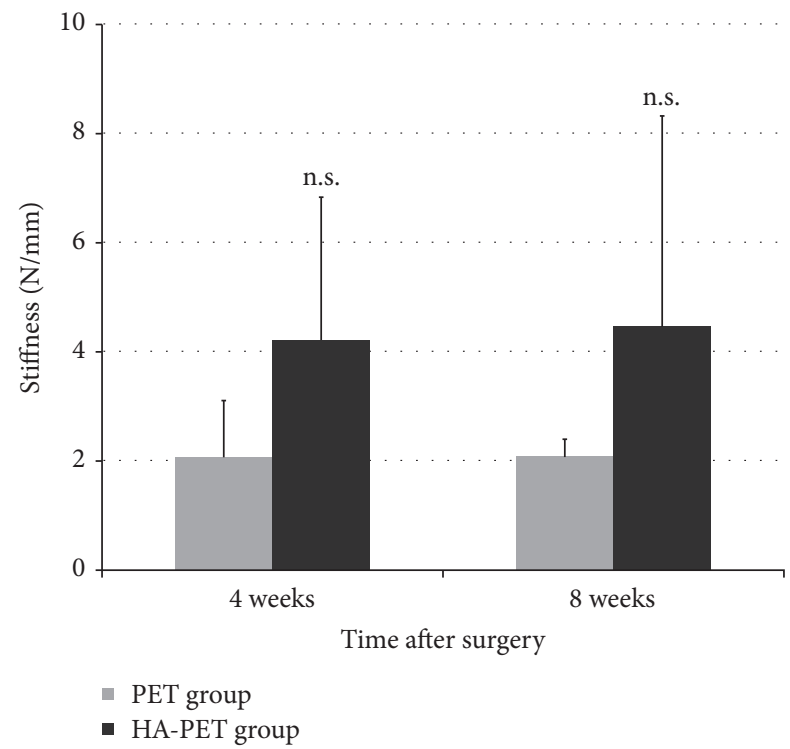

Figure 3: Comparison of stiffness at failure for Achilles tendon healing in a rabbit model of the PET group and HA-PET group at each time after surgery (n.s. indicates there is no significant difference between groups).

\section{Discussion}

Achilles tendon is the most common ruptured tendon of our human body [19]. Reconstruction procedure must be taken using allograft, autograft, or artificial ligament for some serious cases if direct repair is impossible. Artificial ligaments like LARS can provide enough intensity, while it is not fully bioactive for ligamentization. Additional treatment like sodium hyaluronate (HA) can facilitate the process

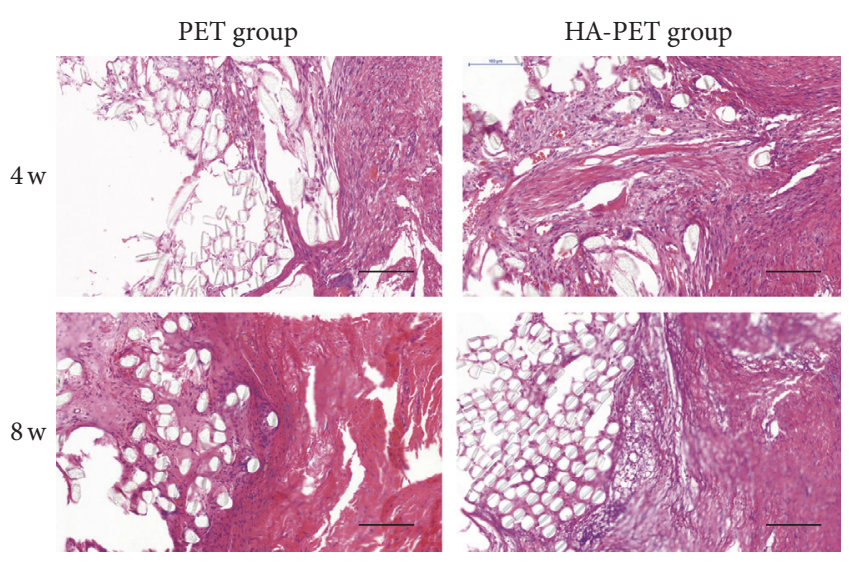

FIGURE 4: Histological images (HE staining) of the PET group and the HA-PET group at 4 weeks and 8 weeks after surgery. Bar = $100 \mu \mathrm{m}$.

of graft healing including inflammation, cellular adhesion and proliferation, vascular proliferation and pruning, and collagen remodeling [20]. In this study, we are trying to find out the effect of sodium hyaluronate for Achilles tendon reconstruction using PET.

Sodium hyaluronate partly contributes to cell adhesion and growth as well as inflammation suppressing. In a previous study, Li et al. found out that polyethylene terephthalate (PET) artificial ligament grafts coated with sodium hyaluronate can enhance cell adhesion, facilitated cell growth, and suppressed the expression of inflammation-related genes relative to a pure PET graft for ACL reconstruction [21]. Another study done by Nakamura et al. demonstrated that HA possesses anti-inflammatory and antiadhesive activities in tendon and synovial fibroblasts derived from RCT [22]. In addition, Salamanna et al. find out that, after repeated peripatellar injections of HA to rats, a significantly higher proliferation rate and viability, along with increased synthesis of C-terminal-propeptide of type I collagen, fibronectin, aggrecan, tenascin-c, and matrix-metalloproteinase- 3 show respect to control group [23]. In this study, the administration of HA improved collagen formation which is good for tendon healing. The molecular weight and the concentration of NaHA influence the rate of the elimination process from the tendon sheath after local injection [24]. There are investigations suggesting that the injection of high-molecular-weight $\mathrm{NaHA}$ in high concentrations around the tendons improves tendon healing and decreases adhesion formation [24-26]. In our study, the weight-average molecular weight of NaHA is within $6 \times 10^{5} \sim 1.5 \times 10^{6}$ which improves tendon healing to a certain extent. There are several different explanations on the effects of NaHA. Some researchers find out that NaHA acts as a physical barrier around the repaired tendon location, while others suggest that the main effect of NaHA may be developed pharmacologically or physiologically [24-26]. However, the exact mechanism of effect of NaHA is not clear.

The histological results especially the Masson staining in this study show that sodium hyaluronate can facilitate new collagen formation. Li et al. suggest that PET graft 


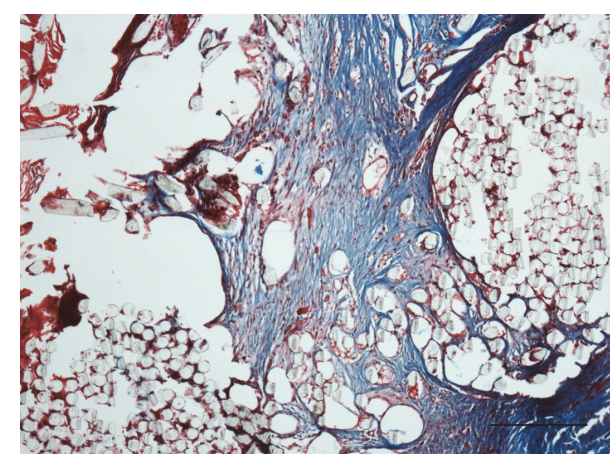

(a)

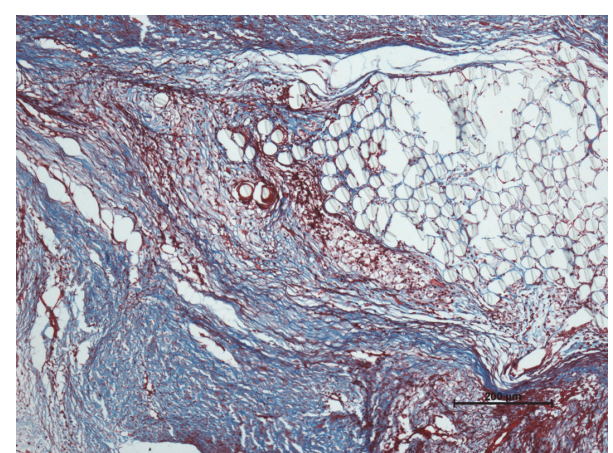

(b)

FIGURE 5: Histological images (Masson staining) of the PET group and the HA-PET group at 8 weeks after surgery. Bar $=200 \mu \mathrm{m}$.

coated with HA can enhance not only cell adhesion and proliferation but also stimulating collagen generation [21]. Oryan et al. demonstrated that, after surgical treatment of tendon rupture, application of sodium hyaluronate to the lesions can improve the density of collagen fibers, almost similar to the normal contra-lateral tendons. Ultrastructural morphometric analyses of the number, diameter (nm), and density (\%) of the collagen fibrils show significant differences with control group on both the number and the diameter of collagen fibrils [27]. In another study Halici et al. suggested that, after 6 weeks of repetitive administration of HA during the rabbit tendon healing process, type IV collagen expression increased significantly, and they believe it is part of the angiogenesis along with the increase of VEGF [28].

In our experiment, the mean load-to-failure and stiffness of HA-PET group appeared higher than those of the PET group. However, there are no significant differences in the mechanical examination results both on the 4 weeks' and 8 weeks' terminal point. Deng et al. [13] investigated the feasibility of using adipose derived stem cells (ASCs) for engineered tendon repair in vivo in a rabbit Achilles tendon model, and they found no difference in tensile strength at 5 weeks but found significant difference at the time points of 12 weeks, 21 weeks, and 45 weeks. Some other studies also show treatment of HA strongly improved the biomechanical properties of the injured tendons and the ultimate strength $[10,27]$. One conceivable possibility is that, unlike with the normal Achilles tendon, PET artificial ligament has much better characteristic of strength and stiffness, and injection of HA may not add significant improvement of biomechanical property to PET artificial ligament. Only 16 rabbits were investigated in our study, which may be somehow insufficient on sample size.

\section{Conclusion}

Collectively, the histological results of our study demonstrate that application of sodium hyaluronate can improve collagen formation, which is beneficial for the healing of Achilles tendon reconstruction with polyethylene terephthalate artificial ligament.

\section{Competing Interests}

The authors report that they have no conflict of interests.

\section{Authors' Contributions}

Shengkun Li and Kui Ma contributed equally to this work.

\section{Acknowledgments}

This project was subsidized by the Natural Science Foundation of China (81401812).

\section{References}

[1] V. Gulati, M. Jaggard, S. S. Al-Nammari et al., "Management of achilles tendon injury: a current concepts systematic review," World Journal of Orthopaedics, vol. 6, no. 4, pp. 380-386, 2015.

[2] R. Arriaza, R. Gayoso, E. López-Vidriero, J. Aizpurúa, and C. Agrasar, "Quadriceps autograft to treat Achilles Chronic tears: a simple surgical technique," BMC Musculoskeletal Disorders, vol. 17, article 116, 2016.

[3] K. Nilsson-Helander, L. Swärd, K. G. Silbernagel, R. Thomeé, B. I. Eriksson, and J. Karlsson, "A new surgical method to treat chronic ruptures and reruptures of the Achilles tendon," Knee Surgery, Sports Traumatology, Arthroscopy, vol. 16, no. 6, pp. 614-620, 2008.

[4] J. M. Deese, G. Gratto-Cox, F. D. Clements, and K. Brown, "Achilles allograft reconstruction for chronic achilles tendinopathy," Journal of Surgical Orthopaedic Advances, vol. 24, no. 1, pp. 75-78, 2015.

[5] M. S. Dhillon, D. Chauhan, V. Kumar, and U. C. Saini, "Reconstruction of bilateral spontaneous chronic idiopathic Achilles tendon rupture using LARS ligament: case report," Foot, vol. 20, no. 4, pp. 154-157, 2010.

[6] C. Legnani, A. Ventura, C. Terzaghi, E. Borgo, and W. Albisetti, "Anterior cruciate ligament reconstruction with synthetic grafts. A review of literature," International Orthopaedics, vol. 34, no. 4, pp. 465-471, 2010.

[7] G. M. Marcheggiani Muccioli, C. Manning, P. Wright, A. Grassi, S. Zaffagnini, and L. Funk, "Acromioclavicular joint reconstruction with the LARS ligament in professional versus non-professional athletes," Knee Surgery, Sports Traumatology, Arthroscopy, vol. 24, no. 6, pp. 1961-1967, 2016. 
[8] E. Circi, Y. E. Akman, E. Sukur, E. R. Bozkurt, T. Tuzuner, and Y. Ozturkmen, "Impact of platelet-rich plasma injection timing on healing of Achilles tendon injury in a rat model," Acta Orthopaedica et Traumatologica Turcica, vol. 50, pp. 366-372, 2016.

[9] M. Sánchez, E. Anitua, J. Azofra, I. Andía, S. Padilla, and I. Mujika, "Comparison of surgically repaired Achilles tendon tears using platelet-rich fibrin matrices," American Journal of Sports Medicine, vol. 35, no. 2, pp. 245-251, 2007.

[10] H. B. Tosun, S. A. Gumustas, M. Kom, A. Uludag, S. Serbest, and Y. Eroksuz, "The effect of sodium hyaluronate plus sodium chondroitin sulfate solution on peritendinous adhesion and tendon healing: an experimental study," Balkan Medical Journal, vol. 33, no. 3, pp. 258-266, 2016.

[11] J. R. E. Fraser, T. C. Laurent, and U. B. G. Laurent, "Hyaluronan: its nature, distribution, functions and turnover," Journal of Internal Medicine, vol. 242, no. 1, pp. 27-33, 1997.

[12] K. R. Kirker, Y. Luo, J. H. Nielson, J. Shelby, and G. D. Prestwich, "Glycosaminoglycan hydrogel films as bio-interactive dressings for wound healing," Biomaterials, vol. 23, no. 17, pp. 3661-3671, 2002.

[13] D. Deng, W. Wang, B. Wang et al., "Repair of Achilles tendon defect with autologous ASCs engineered tendon in a rabbit model," Biomaterials, vol. 35, no. 31, pp. 8801-8809, 2014.

[14] H. Li, Y. Ge, Y. Wu et al., "Hydroxyapatite coating enhances polyethylene terephthalate artificial ligament graft osseointegration in the bone tunnel," International Orthopaedics, vol. 35, no. 10, pp. 1561-1567, 2011.

[15] L. V. Gulotta, D. Kovacevic, L. Ying, J. R. Ehteshami, S. Montgomery, and S. A. Rodeo, "Augmentation of tendon-to-bone healing with a magnesium-based bone adhesive," American Journal of Sports Medicine, vol. 36, no. 7, pp. 1290-1297, 2008.

[16] A. Oryan, A. Moshiri, and A. R. Raayat, "Novel application of theranekron ${ }^{\circledR}$ enhanced the structural and functional performance of the tenotomized tendon in rabbits," Cells Tissues Organs, vol. 196, no. 5, pp. 442-455, 2012.

[17] H. Li, Y. Ge, P. Zhang, L. Wu, and S. Chen, "The effect of layerby-layer chitosan-hyaluronic acid coating on graft-to-bone healing of a poly(Ethylene Terephthalate) artificial ligament," Journal of Biomaterials Science, Polymer Edition, vol. 23, no. 1-4, pp. 425-438, 2012.

[18] J. Buschmann, A. Müller, F. Nicholls et al., "2D motion analysis of rabbits after Achilles tendon rupture repair and histological analysis of extracted tendons: can the number of animals be reduced by operating both hind legs simultaneously?" Injury, vol. 44, no. 10, pp. 1302-1308, 2013.

[19] N. Maffulli, S. W. Waterston, J. Squair, J. Reaper, and A. S. Douglas, "Changing incidence of Achilles tendon rupture in Scotland: A 15-Year Study," Clinical Journal of Sport Medicine, vol. 9, no. 3, pp. 157-160, 1999.

[20] S. M. Joshi, A. N. Mastrangelo, E. M. Magarian, B. C. Fleming, and M. M. Murray, "Collagen-platelet composite enhances biomechanical and histologic healing of the porcine anterior cruciate ligament," American Journal of Sports Medicine, vol. 37, no. 12, pp. 2401-2410, 2009.

[21] H. Li, J. Jiang, Y. Ge et al., "Layer-by-layer hyaluronic acidchitosan coating promoted new collagen ingrowth into a poly(ethylene terephthalate) artificial ligament in a rabbit medical collateral ligament (MCL) reconstruction model," Journal of Biomaterials Science, Polymer Edition, vol. 24, no. 4, pp. 431446, 2013.
[22] H. Nakamura, M. Gotoh, T. Kanazawa et al., "Effects of corticosteroids and hyaluronic acid on torn rotator cuff tendons in vitro and in rats," Journal of Orthopaedic Research, vol. 33, no. 10, pp. 1523-1530, 2015.

[23] F. Salamanna, A. Frizziero, S. Pagani et al., "Metabolic and cytoprotective effects of in vivo peri-patellar hyaluronic acid injections in cultured tenocytes," Connective Tissue Research, vol. 56, no. 1, pp. 35-43, 2014.

[24] L. Hagberg and B. Gerdin, "Sodium hyaluronate as an adjunct in adhesion prevention after flexor tendon surgery in rabbits," Journal of Hand Surgery, vol. 17, no. 5, pp. 935-941, 1992.

[25] S. Işik, S. Öztürk, S. Gürses et al., "Prevention of restrictive adhesions in primary tendon repair by HA-membrane: experimental research in chickens," British Journal of Plastic Surgery, vol. 52, no. 5, pp. 373-379, 1999.

[26] J. A. Miller, R. L. Ferguson, D. L. Powers, J. W. Burns, and S. W. Shalaby, "Efficacy of hyaluronic acid/nonsteroidal antiinflammatory drug systems in preventing postsurgical tendon adhesions," Journal of Biomedical Materials Research, vol. 38, no. 1, pp. 25-33, 1997.

[27] A. Oryan, A. Moshiri, and A.-H. Meimandiparizi, "Effects of sodium-hyaluronate and glucosamine-chondroitin sulfate on remodeling stage of tenotomized superficial digital flexor tendon in rabbits: a clinical, histopathological, ultrastructural, and biomechanical study," Connective Tissue Research, vol. 52, no. 4, pp. 329-339, 2011.

[28] M. Halici, S. Karaoglu, O. Canoz, S. Kabak, and A. Baktir, "Sodium hyaluronate regulating angiogenesis during Achilles tendon healing," Knee Surgery, Sports Traumatology, Arthroscopy, vol. 12, no. 6, pp. 562-567, 2004. 


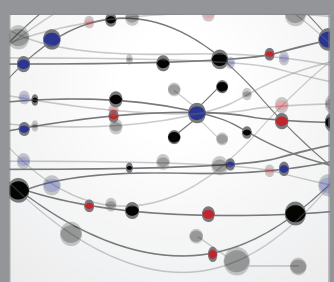

The Scientific World Journal
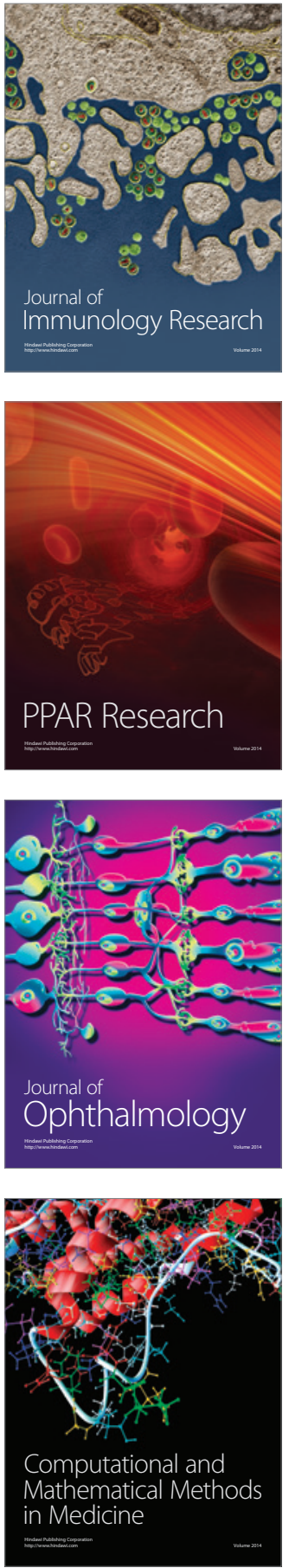

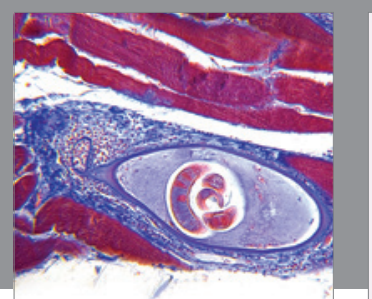

Gastroenterology Research and Practice

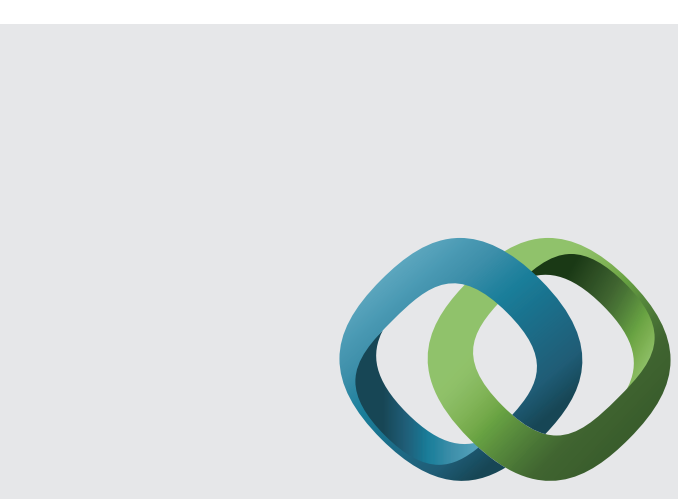

\section{Hindawi}

Submit your manuscripts at

http://www.hindawi.com
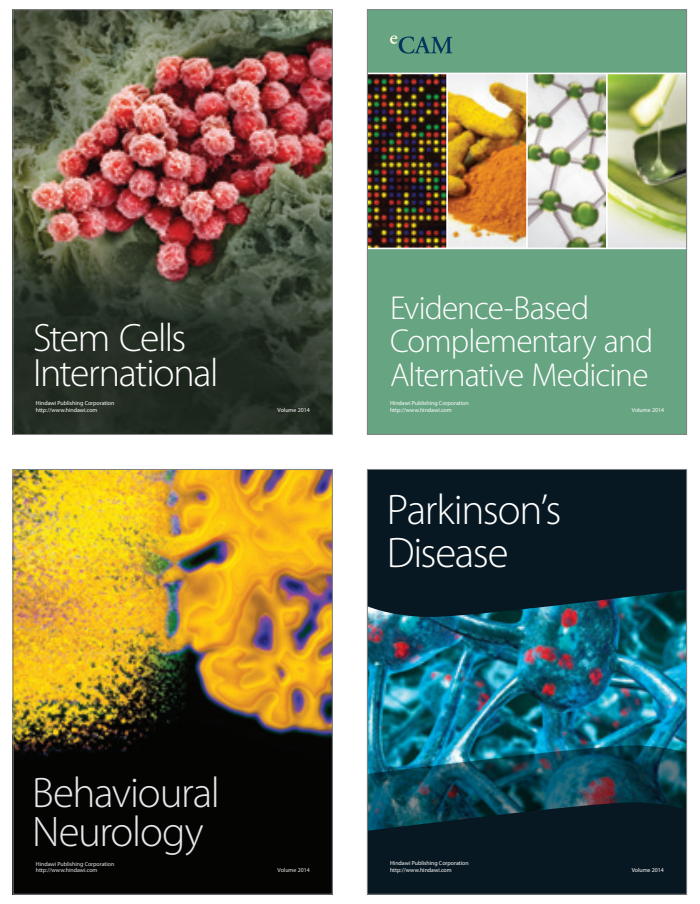
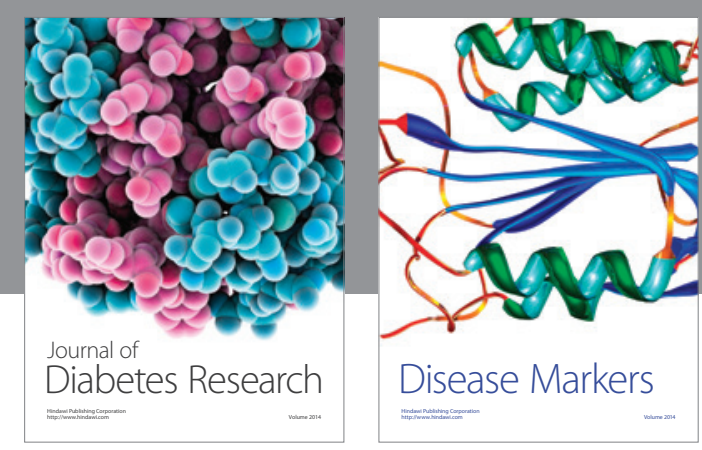

Disease Markers
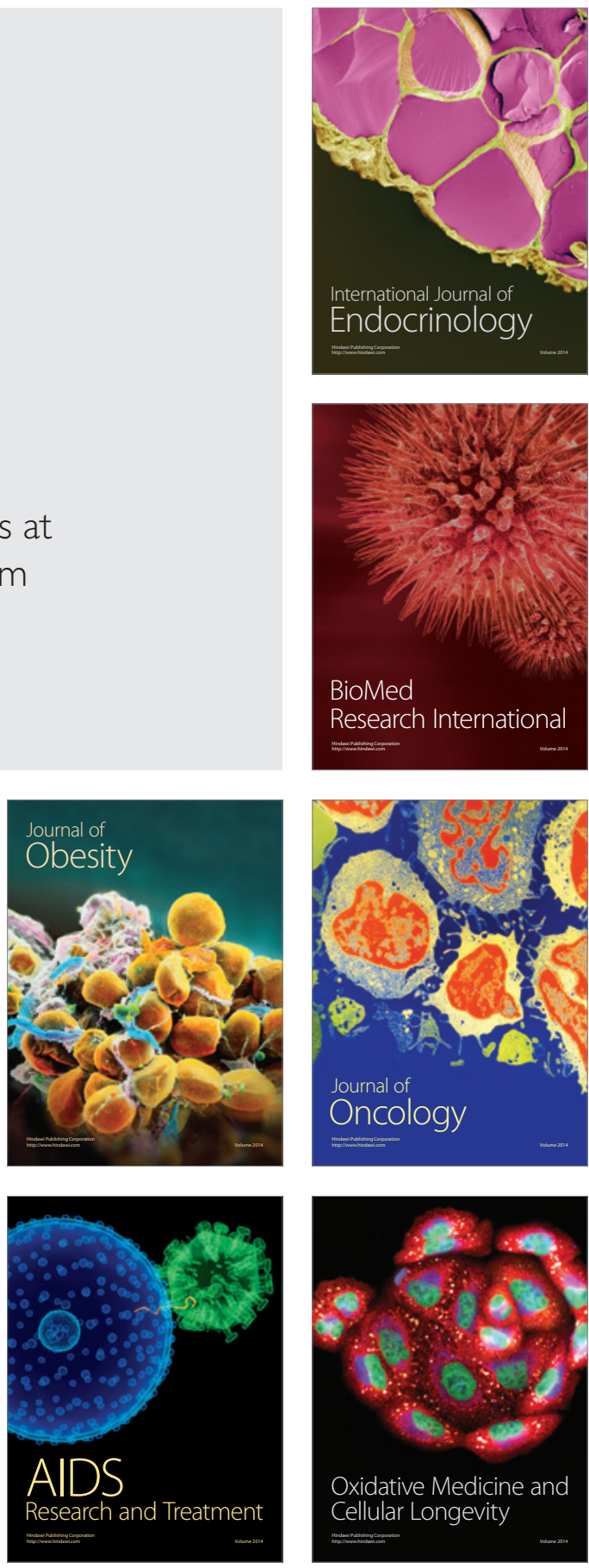